\title{
Summary of: NICE guideline and current practice of antibiotic prophylaxis for high risk cardiac patients (HRCP) among dental trainers and trainees in the United Kingdom (UK)
}

FULL PAPER DETAILS

1"Department of Oral and Maxillofacial Surgery, University College Hospital, 235 Euston Road, London NW1 2BU; ${ }^{2}$ Department of Oral and Maxillofacial Surgery, Queen Victoria Hospital, Holtye Road, East Grinstead, West Sussex, RH19 3DZ; 3Primary Dental Care, King's College Hospital, Denmark Hill, London, SE5 9RS; ${ }^{4}$ Department of Cardiothoracic Surgery, The Heart Hospital, 16-18 Westmoreland Street, London, W1G $8 \mathrm{PH}$

${ }^{*}$ Correspondence to: Mr S. A. Farook

Email:safarook@hotmail.com; Tel: 01342414000

Refereed Paper

Accepted 25 May 2012

DOI: 10.1038/sj.bdj.2012.723

${ }^{\circ}$ British Dental Journal 2012; 213: E6

\author{
S. A. Farook, ${ }^{1}$ A. K. J. Davis, ${ }^{2}$ N. Khawaja ${ }^{3}$ and A. M. Sheikh ${ }^{4}$
}

VERIFIABLE CPD PAPER

Objective The National Institute for Health and Clinical Excellence (NICE) introduced the antibiotic prophylaxis guideline in 2008 for cardiac patients in the UK, which has led to a decrease in national prescription levels for antibiotic prophylaxis. Despite the introduction of the guideline there is still a discrepancy in levels of compliance among the dental community. The aims of this study were to determine the understanding of the NICE clinical guideline on antimicrobial prophylaxis against infective endocarditis (IE) and the difference in antibiotic prescription for high risk cardiac patients (HRCP) between dental trainers and trainees. Methods A proforma was designed and distributed among dental trainers and trainees attending a conference at the London deanery. The trainers were GDPs responsible for training dental trainees allocated to them over a 12 month period based in a general dental practice. Dental trainees were recent graduates about to commence their vocational dental training. Eighty-five vocational dental trainees and 70 trainers completed the proforma on a voluntary basis. Results The results of the study confirm that most trainers (95.7\%) and trainees (94.1\%) are aware of this guideline but only $62 \%$ of trainers and $69.7 \%$ of trainees have read the guideline. Compliancy with the guideline was low among trainers (55.7\%) and trainees (77.6\%). Compliance was high among those who had read the guideline. Trainers were more likely to prescribe prophylaxis antibiotics for HRCP. The majority (74-76\%) would prescribe antibiotics on a specialist's request. Some trainers (54.9\%) and trainees (48.2\%) would want antibiotics themselves if they were HRCP. Conclusion This study concludes that much needs to be done to improve the understanding and practice of NICE guideline among the dental trainers and trainees.

\section{EDITOR'S SUMMARY}

Damned if we do and almost damned if we don't. The debate over whether or not to provide antibiotic prophylaxis to patients with heart conditions should to all intents and purposes have been stiffled by the publication in 2008 of the NICE guidelines. However, it seems that it is not as simple as that.

To begin with, the NICE guidance is at odds with that issued by other authorities internationally, one of whose criticisms is based on the fact that the background is a literature search rather than 'proof'. On the other hand, since the guidelines were introduced and antibiotic prescription has fallen by $78.6 \%$ there have been no notable effect on the identified cases of infective endocarditis (IE) in England, to which one might ask; how much proof do you need?
This piece of research, while touching on these dilemmas also helps examine the moral, or perhaps, hierarchical issues which also come into play in dealing with this clinical eventuality. Should a trainee question the instructions of a trainer, should a GDP similarly question a GP or a specialist? The law seems clear, in that should a patient suffer a negative consequence of a dentist not providing antibiotic cover, presumably in the form of IE, then he or she would be fully defensible for having followed current (ie NICE) guidelines.

What none of us would want though is an adverse patient experience that could be prevented and into the melee of complexities comes a further twist in the handling of patients who have previously received cover. When told that this is no longer required they obviously need a very detailed and thorough explanation as to the reasons for the change. To this end it is pertinent that in this study $54.9 \%$ and $48.2 \%$ of trainers and trainees respectively reported that they would still wish to have antibiotic cover if they were a high risk cardiac patient.

Clearly there needs to be considerably more debate on the matter and greater understanding of the evidence base, or at least value of the literature, in arriving at the safest and most beneficial route for optimum patient care.

The full paper can be accessed from the $B D J$ website (www.bdj.co.uk), under 'Research' in the table of contents for Volume 213 issue 4.

Stephen Hancocks Editor-in-Chief

DOI: 10.1038/sj.bdj.2012.752 
TO ACCESS THE BDJ WEBSITE TO READ THE FULL PAPER:

- BDA Members should go to www.bda.org.

- Click the 'login' button on the right-hand side and enter your BDA login details.

- Once you have logged in click the 'BDJ' tab to transfer to the BDJ website with full access.

IF YOUR LOGIN DETAILS DO NOT WORK:

- Get a password reminder: go to www.bda.org, click the login button on the right-hand side and then click the forgotten password link.

- Use a recommended browser: we recommend Microsoft Internet Explorer or Mozilla Firefox.

- Ensure that the security settings on your browser are set to recommended levels.

IF YOU HAVE NOT YET SIGNED UP TO USE THE BDA WEBSITE:

- Go to www.bda.org/getstarted for information on how to start using the BDA website.
IN BRIEF

- Highlights the differences between the NICE guideline for antibiotic prophylaxis against infective endocarditis and guidelines from other parts of the world.

- Defines and describes the high risk cardiac patient group.

- Highlights the legal implications applicable to the NICE guideline.

\section{COMMENTARY}

Modern airliners are designed to be flown by two people with one pilot checking what the other is doing and correcting errors. The airline industry's analysis of crashes indicates that if the senior pilot is at the controls, the junior pilot is often not willing to correct their senior's errors; there is a greater risk of an accident with the more experienced pilot flying the plane. ${ }^{1}$

In a similar way, dental foundation trainees sometimes find it difficult not to comply with the protocols that their trainers implement in the training practice. Trainees find it even more difficult to contradict a GP or cardiologist who insists that antibiotic prophylaxis should be prescribed to patients at risk of infective endocarditis. The patient might also insist, and it takes a skilled response to reassure and inform patients, who have always had antibiotic cover, of the latest evidence-based practice.

Trainers have an important part to play in guiding, educating and sometimes correcting young dentists in their first year of professional practice. The way that a trainer acts can have a great influence on their trainee, for good or ill. A trainee can derive power from a well-informed trainer to say no to something they know is not best practice, whoever is trying to influence them.

It is incumbent upon all involved in foundation training to ensure that our acts are based upon the best current evidence. In this case the National Institute for Health and
Clinical Excellence carefully reviewed the literature and decided that the balance of risk was against giving antibiotic cover.

\section{Dr K J Cottingham}

Regional Director Dental

Foundation Training (year one)

South Yorkshire/East Midlands

Postgraduate Dental Deanery,

Don Valley House,

Savile St, East Sheffield, S4 7UO

1. Gladwell M. The ethnic theory of plane crashes. In Outliers: the story of success. London: Penguin Books Ltd, 2009.

\section{AUTHOR QUESTIONS AND ANSWERS}

1. Why did you undertake this research? NICE guidelines for antibiotic prophylaxis against infective endocarditis (IE) for patients undergoing dental procedures has been a regular point of discussion among the dental community. Therefore, this study was undertaken to determine the awareness, understanding and the practice of antibiotics prescription among dental trainers and trainees who are a cohort group among the dental professionals expected to have increased awareness of such guidelines and practice. Furthermore, we also wanted to determine how they would respond to a request from a patient or a medical professional and what they would want themselves if deemed a high risk cardiac patient.

2. What would you like to do next in this area to follow on from this work?

We would like to carry out a study among the cardiologists and cardiothoracic surgeons to determine their understanding and their views on NICE guidelines for antibiotic prophylaxis against infective endocarditis. 